\title{
Authors' Reply to Eerdekens et al. “Treating Pain in Diabetic Neuropathy: Current and Developmental Drugs"
}

\author{
Uazman Alam ${ }^{1,2,3} \cdot$ Gordon Sloan $^{4} \cdot$ Solomon Tesfaye ${ }^{4,5}$
}

Published online: 4 July 2020

○) Springer Nature Switzerland AG 2020

\section{Dear Editor,}

We thank Eerdekens et al. [1] for their detailed review and comments in relation to our manuscript published in Drugs [2]. We acknowledge that neuropathic pain represents a tremendous challenge worldwide and high concentration capsaicin patch (8\%) [HCCP] may be a viable option for a subgroup of patients with painful diabetic neuropathy (pDPN). Indeed, HCCP is FDA-approved for post herpetic neuralgia (2009) and EMA-approved for pDPN (2015), and represents an alternative to systemic (oral) therapy that can often result in intolerable adverse effects resulting in treatment withdrawal. We agree topical analgesic treatments are generally underutilized [3] and the treatment of pDPN can be challenging in the elderly who have an increased risk of adverse effects through systemic polypharmacy. Indeed, topical therapy of which only HCCP is approved for the treatment of pDPN represents a valid alternative, providing a superior adverse effect profile. A network meta-analysis by van Nooten et al. [4] suggested similar efficacy of HCCP compared with oral agents (pregabalin, duloxetine, gabapentin) in patients with pDPN. The authors concluded that HCCP "was as effective as oral centrally acting agents in

Uazman Alam

Uazman.alam@manchester.ac.uk

1 Institute of Cardiovascular and Metabolic Medicine and The Pain Research Institute, University of Liverpool, Liverpool, UK

2 Division of Endocrinology, Diabetes and Gastroenterology, University of Manchester, Manchester, UK

3 Department of Diabetes and Endocrinology, Liverpool University Hospital NHS Foundation Trust, Liverpool, UK

4 Diabetes Research Unit, Royal Hallamshire Hospital, Sheffield Teaching Hospitals NHS Foundation Trust, Sheffield, UK

5 Department of Oncology and Human Metabolism, University of Sheffield, Sheffield, UK these patients with pDPN but offers systemic tolerability benefits" [4].

A robust Cochrane Collaboration review (2016) of HCCP in postherpetic neuralgia, HIV neuropathy, and pDPN did suggest more participants received benefit with moderate or substantial levels of pain relief with HCCP compared with a lower concentration of capsaicin [5]. Unfortunately, the quality of the evidence was moderate to very low for pain relief outcomes, due to the small number of studies and moderate number of participants [5]. HCCP was considered similar in its effects to other therapies for chronic pain [5]. In addition, Neuropathic Pain Special Interest Group (NeuPSIG) guidelines considered the use of HCCP as second line [6]. The number needed to treat for benefit (NNTB) for 50\% pain relief was 10.6 (95\% confidence interval: 7.4-19), which is greater than other first- and second-line therapies [6]. The quality of evidence for HCCP was considered 'high', with a good balance of desirable to undesirable adverse effects; however, the strength of recommendations was 'weak' (in favor) based on the Grading of Recommendations Assessment, Development, and Evaluation (GRADE) classification [6]. Data for HCCP were also most likely to be subject to publication bias [6]. The current American Diabetes Association guidelines for diabetic neuropathy (2017) do not advocate the use of HCCP or low-dose capsaicin cream for the treatment of pDPN; however, guidelines from the German Association of Neurology (2019) [7] recommend HCCP for focal treatment of neuropathic pain. Eerdekens et al. [1] have correctly stated that National Institute for Health and Care Excellence (NICE) guidance (CG173) [8] is specific to a non-specialist setting and HCCP is primarily used in specialist care. Due to procedural requirements in the application of HCCP, which may include premedication with opiates and prior application of topical lidocaine, HCCP is likely to remain in use in the specialist care setting. Post application adverse effects include 48-72 $\mathrm{h}$ of pain at the site of application, often described as 'deep heat, like a bad sunburn' [9]. 
We welcome the recount of Simpson et al. [10], which highlights the efficacy of HCCP in pDPN; however, there are notable limitations to that study. Although there was no indication of deterioration of sensory function, the method sensory of phenotyping in our opinion was less than optimal [10]. Unfortunately, neither the study by Simpson et al. [10] nor the study by Vinik et al. [11] utilized the gold standard sensory testing paradigm (German Research Network on Neuropathic Pain [DFNS]) [12]. A study of healthy volunteers has exhibited sensory dysfunction with thermal threshold testing after HCCP application at 6 months [13]. We understand the concerns regarding the lack of adverse event reporting of oral therapies in the abstract; however, crucially, none of the first- or second-line oral drugs for pDPN, e.g. pregabalin, duloxetine, gabapentin and amitriptyline, are known to cause degeneration of sensory nerve fibers. Polydefkis et al. [14] highlighted the ability of $0.1 \%$ capsaicin to totally denervate the skin, with reduced regenerative capacity in diabetes and even greater in pDPN. In fact, there is a reduction in the rate of regeneration in the presence of diabetes compared with healthy volunteers. Furthermore, those with neuropathy had a reduced regenerative rate compared with those without neuropathy [14]. Importantly, the rate of regeneration was dependent on the baseline epidermal nerve fiber density [14]. A study of HCCP applied to the inner thigh at the recommended dose (a single application for $60 \mathrm{~min}$ ) produced approximately $60 \%$ epidermal denervation [15]. Gibbons et al. [13] undertook a detailed skin biopsy study of $0.1 \%$ capsaicin cream (or placebo) for $48 \mathrm{~h}$ applied via an occlusive dressing in healthy volunteer subjects $(n=32)$. There was clear degeneration of sudomotor, vasomotor, pilomotor and sensory nerve fiber densities with a concomitant dysfunction of each fiber type [13]. Autonomic nerve fibers regenerated to baseline levels at 40-50 days, while sensory fibers required $140-150$ days for regeneration [13]. Given that HCCP may be applied every 90 days, sensory nerve fibers may theoretically not recover to baseline levels with repeated application. Gibbons' et al. [13] interpretation of their data is "caution should be taken when topical capsaicin is applied to skin surfaces at risk for ulceration, particularly in neuropathic conditions characterized by sensory and autonomic impairment".

Showing reversible degeneration (Chiang et al. [16]) and possible regeneration (Anand et al. [17]) in healthy volunteers and chemotherapy-induced peripheral neuropathy (CIPN), respectively, is reassuring but only in relation to these groups. Numerous studies, including our data, have shown lower intraepidermal nerve fiber density (IENFD) in diabetes groups, even without neuropathy, suggesting preclinical pathology [18]. Unlike CIPN, the metabolic insult to the small nerve fibers is lifelong in diabetes, therefore direct comparisons of regeneration after HCCP in these disease/ non-disease groups is problematic. At a population level,
pDPN prevalence increases with the increasing severity of diabetic neuropathy [19]. Anecdotally, the use of HCCP in pDPN is predominantly in those with more severe diabetic neuropathy with intractable pain. Given that diabetic neuropathy is the major contributor to diabetic foot ulceration, which has a woeful 5-year mortality, exacerbation of sensory, vasomotor and sudomotor dysfunction, key mechanisms in the development of diabetic foot disease, should be avoided.

Unfortunately, studies of HCCP in pDPN (including NCT01478607, NCT04238208, NCT01533428) have not undertaken skin biopsy to determine the effects on IENFD or sensory dysfunction in the long term. However, the Capsaicin Pain Patch study (EudraCT number: 2017-00474617), a randomized parallel trial of HCCP versus standard of care (ratio 2:1) for 52 weeks with 12 weekly applications of HCCP and skin biopsies for intraepidermal nerve fibers, is currently underway. In addition, bedside sensory testing is included in the scientific protocol. The results of this study are eagerly awaited and a positive result in terms of nerve fiber regeneration in the presence of repeated longterm HCCP application will provide health care professionals with significant reassurance. Indeed, it may allow for greater use of HCCP within secondary care, specifically by endocrinologists with the development of specialist services.

\section{Compliance with Ethical Standards}

Conflict of interest Uazman Alam has received honoraria for educational meetings from Sanofi, Napp, Boehringer Ingelheim, Pfizer and Eli Lilly, and is currently a local investigator for BIIB074 (Vixotrigine) [Biogen: NCT03339336]. Solomon Tesfaye reports grants from Impeto Medical; personal fees from Neurometrix, Pfizer, Miro, Worwag Pharma, Mundipharma, Merck and Mitsubishi Pharma; and personal fees and other from Novo Nordisk. Gordon Sloan has no conflicts of interest to declare.

\section{References}

1. Eerdekins M, Stupar M, Marcondes L. Comment on treating pain in diabetic neuropathy: current and developmental drugs. Drugs. 2020. https://doi.org/10.1007/s40265-020-01353-5.

2. Alam U, Sloan G, Tesfaye S. Treating pain in diabetic neuropathy: current and developmental drugs. Drugs. 2020;80(4):363-84.

3. Peppin JF, Pappagallo M. Capsaicinoids in the treatment of neuropathic pain: a review. Ther Adv Neurol Disord. 2014;7(1):22-32.

4. van Nooten F, et al. Capsaicin $8 \%$ patch versus oral neuropathic pain medications for the treatment of painful diabetic peripheral neuropathy: a systematic literature review and network metaanalysis. Clin Ther. 2017;39(4):787-803.e18.

5. Derry S, Rice AS, Cole P, Tan T, Moore RA. Topical capsaicin (high concentration) for chronic neuropathic pain in adults. Cochrane Database Syst Rev. 2017;(1):CD007393.

6. Finnerup NB, et al. Pharmacotherapy for neuropathic pain in adults: a systematic review and meta-analysis. Lancet Neurol. 2015;14(2):162-73. 
7. Schlereth T. Guideline, "diagnosis and non interventional therapy of neuropathic pain" of the German Society of Neurology (deutsche Gesellschaft für Neurologie). Neurol Res Pract. 2020;2(1):16.

8. National Institute for Health and Care Excellence. Neuropathic pain in adults: pharmacological management in non-specialist settings [CG173]. National Institute for Health and Care Excellence; 2013.

9. Baranidharan G, Das S, Bhaskar A. A review of the highconcentration capsaicin patch and experience in its use in the management of neuropathic pain. Ther Adv Neurol Disord. 2013;6(5):287-97.

10. Simpson DM, et al. Capsaicin $8 \%$ patch in painful diabetic peripheral neuropathy: a randomized, double-blind, placebo-controlled study. J Pain. 2017;18(1):42-53.

11. Vinik AI, et al. Capsaicin $8 \%$ patch repeat treatment plus standard of care (SOC) versus SOC alone in painful diabetic peripheral neuropathy: a randomised, 52-week, open-label, safety study. BMC Neurol. 2016;16(1):251.

12. Rolke R, et al. Quantitative sensory testing in the German Research Network on Neuropathic Pain (DFNS): standardized protocol and reference values. Pain. 2006;123(3):231-43.
13. Gibbons CH, Wang N, Freeman R. Capsaicin induces degeneration of cutaneous autonomic nerve fibers. Ann Neurol. 2010;68(6):888-98.

14. Polydefkis M, et al. The time course of epidermal nerve fibre regeneration: studies in normal controls and in people with diabetes, with and without neuropathy. Brain. 2004;127(Pt 7):1606-15.

15. Malmberg AB, et al. Reduced heat sensitivity and epidermal nerve fiber immunostaining following single applications of a high-concentration capsaicin patch. Pain. 2004;111(3):360-7.

16. Chiang $\mathrm{H}$, et al. Physiological and pathological characterization of capsaicin-induced reversible nerve degeneration and hyperalgesia. Eur J Pain. 2018;22(6):1043-56.

17. Anand $\mathrm{P}$, et al. Rational treatment of chemotherapy-induced peripheral neuropathy with capsaicin $8 \%$ patch: from pain relief towards disease modification. J Pain Res. 2019;12:2039-52.

18. Alam U, et al. Diagnostic utility of corneal confocal microscopy and intra-epidermal nerve fibre density in diabetic neuropathy. PLoS ONE. 2017;12(7):e0180175.

19. Abbott CA, et al. Prevalence and characteristics of painful diabetic neuropathy in a large community-based diabetic population in the U.K. Diabetes Care. 2011;34(10):2220-4. 